\title{
Anacetrapib as a potential cardioprotective strategy
}

This article was published in the following Dove Press journal:

Drug Design, Development and Therapy

\section{Belinda A Di Bartolo Stephen J Nicholls}

South Australian Health and Medical Research Institute, University of Adelaide, Adelaide, SA, Australia
Correspondence: Stephen J Nicholls South Australian Health and Medical Research Institute, PO Box I 1060 , Adelaide, SA 500I, Australia

Tel +6I 881284510

Email stephen.nicholls@sahmri.com

\begin{abstract}
Cholesteryl ester transfer protein (CETP) facilitates movement of esterified cholesterol between high-density lipoproteins (HDLs) and apolipoprotein B-containing lipoproteins. By virtue of their ability to raise HDL cholesterol and lower low-density lipoprotein cholesterol, pharmacological inhibitors of CETP have received considerable attention as potential new agents in cardiovascular prevention. While early studies of CETP inhibitors have demonstrated a lack of clinical efficacy and potential toxicity, development of the potent CETP inhibitor, anacetrapib, has moved forward, with emerging evidence suggesting a role in reducing cardiovascular events. The experience with anacetrapib and its potential for use in clinical practice are reviewed here.
\end{abstract}

Keywords: anacetrapib, CETP, lipids, atherosclerosis, cardiovascular disease

\section{Introduction}

Consistent findings of cardiovascular benefit with statins have led to their widespread use in clinical practice. However, there remains a considerable residual risk of clinical events suggesting the need to develop additional therapeutic strategies that will further reduce cardiovascular risk in the statin-treated patients. In addition, many patients have difficulty tolerating statins at doses required to achieve the degree of lipid lowering considered appropriate in cardiovascular prevention guidelines. There has been interest in developing approaches that will achieve more effective lowering of low-density lipoprotein cholesterol (LDL-C), the atherogenic lipid parameter associated with cardiovascular risk. In addition, there have been major efforts to develop therapies that elevate high-density lipoprotein cholesterol (HDL-C), which is thought to protect against the development of atherosclerotic cardiovascular disease.

\section{CETP}

Cholesteryl ester transfer protein (CETP) is a plasma-based factor that plays an important role in lipid metabolism, by facilitating exchange of esterified cholesterol from high-density lipoprotein (HDL) to very low-density lipoprotein (VLDL) and lowdensity lipoprotein (LDL). ${ }^{1}$ It remains to be determined whether CETP acts primarily by forming a tunnel between lipoproteins or acts as a shuttle, in order to move lipid species between particles. Enthusiasm for developing pharmacological inhibitors was supported by observations that raising HDL-C and lowering LDL-C ${ }^{2}$ exert favorable effects on atherosclerotic plaque in rabbit models ${ }^{3-6}$ and that polymorphisms associated with low CETP activity lead to lower cardiovascular risk. ${ }^{1,7}$ While numerous approaches have been developed to reduce CETP activity, including oligosense antinucleotides and vaccines, it has been the development of small-molecule inhibitors that has received the most attention..$^{3-6}$ 


\section{Experience with prior CETP inhibitors}

The early experience with CETP inhibitors that have progressed to an advanced phase of clinical development has been disappointing (Table 1). Torcetrapib was the first agent to proceed to a large cardiovascular outcomes trial. However, this study was terminated prematurely due to the finding of an excess in mortality and cardiovascular events. This occurred despite HDL-C raising by more than $60 \%$ and incremental lowering of LDL-C by $20 \%$ in statin-treated patients. ${ }^{9}$ In parallel, three serial vascular imaging studies failed to demonstrate any beneficial effect of torcetrapib in slowing progression of either carotid intima-medial thickness or coronary atherosclerosis. ${ }^{10-12}$

This disappointing finding prompted concerns that CETP inhibition might have adverse effects on HDL function. However, a number of observations suggested that HDL functionality was intact with torcetrapib. HDL continues to promote cholesterol efflux in the setting of CETP deficiency and torcetrapib treatment. ${ }^{13}$ Furthermore, plaque regression and lower cardiovascular event rates were observed in torcetrapib-treated patients achieving the highest HDL-C levels. ${ }^{14,15}$ The findings that torcetrapib increased adrenal synthesis of aldosterone and cortisol, and upregulated aortic wall endothelin expression, suggested that torcetrapib possessed off-target toxicities. ${ }^{9,15,16}$ These findings paved the way for the development of other CETP inhibitors that lacked such off-target effects.

Dalcetrapib is a less potent CETP inhibitor, modestly raising HDL-C by $30 \%$ without lowering LDL-C. Phase II studies demonstrated that dalcetrapib had no torcetrapib-associated off-target effects and no adverse effects on endothelial function or arterial wall inflammation. ${ }^{17,18}$ However, a large outcomes trial, performed in patients treated from 8 weeks following an acute coronary syndrome, demonstrated no effect on cardiovascular events. ${ }^{19}$ Subsequent studies have revealed that patients harboring the ADCY9 polymorphism demonstrated favorable effects on cholesterol efflux activity and lesser increases in inflammatory markers associated with a favorable reduction in cardiovascular events and progression of carotid intima-medial thickness. ${ }^{20}$ This has led to the performance of an innovative clinical trial, in which patients harboring this polymorphism will be prospectively randomized to treatment with dalcetrapib or placebo, in order to determine whether this pharmacogenomic approach to patient selection will result in a clinically meaningful benefit in terms of event reduction.

Evacetrapib is a potent CETP inhibitor, which produced increases in HDL-C greater than 125\% and LDL-C lowering by more than $30 \%$, as monotherapy or in addition to commonly prescribed doses of statin therapy. ${ }^{21}$ However, the large cardiovascular outcomes trial of this agent was terminated due to lack of clinical benefit. ${ }^{22}$ Despite favorable lipid changes and no evidence of torcetrapib-associated toxicity, there was no patient subgroup that demonstrated a reduction in cardiovascular events. ${ }^{22}$ Additional lipid studies demonstrated increases in C-reactive protein and apolipoprotein (apo) E and apoC-III, correlating with changes in HDL-C. ${ }^{23}$ Whether the lack of clinical benefit despite favorable lipid effects reflects a potential proinflammatory state or ability of larger HDL particles to carry atherogenic factors such as apoC-III remains to be determined. Of particular interest, in all of these studies of CETP inhibitors to date, ex vivo measures of cellular cholesterol efflux appeared to increase in association with treatment with a CETP inhibitor. ${ }^{23}$ As a result, there is considerable confusion as to why CETP inhibitors have not produced cardiovascular benefit to date.

\section{Anacetrapib}

Anacetrapib is a lipophilic and potent CETP inhibitor, which has been extensively investigated across the translational spectrum. In addition to robust increases in HDL-C levels,

Table I Properties of CETP inhibitors that have proceeded to advanced stages of clinical development

\begin{tabular}{|c|c|c|c|c|c|}
\hline Parameter & Torcetrapib & Dalcetrapib & Evacetrapib & Anacetrapib & TA-8995 \\
\hline Molecular weight $(\mathrm{Da})$ & 600.4 & 389.6 & 638.6 & 673.5 & 722.6 \\
\hline $\begin{array}{l}\text { Adipose tissue } \\
\text { accumulation }\end{array}$ & None & None & None & $\begin{array}{l}\text { Demonstrated with } \\
\text { terminal half-life }>\text { I year }\end{array}$ & None \\
\hline HDL-C & $+70 \%$ & $+30 \%$ & $+125 \%$ & $+130 \%$ & $>+76 \%$ \\
\hline LDL-C & $-20 \%$ & $0 \%$ & $-25 \%$ & $-25 \%$ & $>-27 \%$ \\
\hline Cholesterol efflux & Increase & Increase & Increase & Increase & Increase \\
\hline Blood pressure & $3-6 \mathrm{mmHg}$ increase & No effect & No effect & No effect & No effect \\
\hline Clinical outcomes & $\begin{array}{l}\text { Adverse effects on } \\
\text { mortality and } C V \text { events }\end{array}$ & Clinical futility & Clinical futility & $\begin{array}{l}9 \% \text { reduction in } \mathrm{CV} \\
\text { events }\end{array}$ & Unknown \\
\hline
\end{tabular}

Abbreviations: CETP, cholesteryl ester transfer protein; HDL-C, high-density lipoprotein cholesterol; LDL-C, low-density lipoprotein cholesterol; CV, cardiovascular. 
anacetrapib also produces concentration-dependent lowering of LDL-C, apoB and lipoprotein(a) (Lp(a)). ${ }^{24}$ These effects have been consistently demonstrated in preclinical and human studies, which have formed the basis of the evaluation of its impact on cardiovascular outcomes in a large, randomized clinical trial. The confidence to pursue this clinical development program was derived from studies that suggested that anacetrapib was unlikely to possess the toxic effects observed with torcetrapib. In addition to establishing the efficacy and safety profile, studies of anacetrapib have enabled characterization of the impact of potent CETP inhibitors on the factors regulating lipid metabolism.

\section{Experience from animal studies}

The use of animal models in studies of CETP inhibitors is challenged by the observation that mouse models commonly employed in the study of atherosclerosis do not endogenously express CETP. In contrast, rabbits constitutively express CETP in a similar manner to humans. Mouse models have been important in their ability to demonstrate that the adverse effects of torcetrapib on blood pressure and the vasculature are observed in the absence of CETP, supporting the likelihood that this reflects an off-target effect. ${ }^{25}$ Genetic expression of CETP, using the ApoE*3Leiden. CETP-TG mouse, has enabled study of the impact of anacetrapib treatment on atherosclerosis, with evidence of dose-dependent favorable effects on lesion size and composition, whether administered as monotherapy or in combination with atorvastatin. ${ }^{26}$ This was observed, despite additional reports that anacetrapib treatment was associated with impairment in endothelial function in this model. ${ }^{27}$ More recent examinations have revealed prolonged storage of anacetrapib within white adipose tissue, with only minimal reduction in adipose concentrations more than 1 year following administration. ${ }^{28}$ This has been important to understand as there has been ongoing work to study the long-term disposition of anacetrapib following administration. Studies in the Syrian golden hamster have demonstrated an increase in measures of in vivo reverse cholesterol transport with anacetrapib. ${ }^{29}$ Whether this contributes to potential anti-atherosclerotic effects is unknown.

Additional studies performed in the rabbit model have demonstrated contrasting results with regard to the effect of anacetrapib on the endothelium. Studies within the aorta demonstrated that anacetrapib treatment enhances endothelial repair following denudation injury and improves endothelial function, ${ }^{30}$ in contrast to observations in mouse models. In a hindlimb ischemia model, anacetrapib treatment was associated with an increase in collateral blood vessel formation, in a process that is dependent on the scavenger receptor, SR-BI, and PI3K/Akt pathways. ${ }^{31}$ This suggests a potentially favorable effect on angiogenesis.

\section{Experience from metabolic studies}

Increasing use of stable isotopes has permitted investigation of production and catabolism of individual lipoproteins, with the opportunity to characterize the impact of a range of disease settings and therapeutic interventions. Using these techniques, a number of important insights into the impact of anacetrapib, and therefore potentially potent CETP inhibition, on lipoprotein metabolism have been made. Increases in HDL-C and apoA-I result from a reduction in the fractional catabolic rate of each parameter, without any discernible change in production. ${ }^{32}$ In contrast, the observed reduction in apoB and LDL-C results from an increase in the fractional catabolic rate of apoB. ${ }^{33}$ Similarly, an increase in the fractional catabolic rate of VLDL triglyceride appears to underscore observed reductions in triglyceride levels with anacetrapib. ${ }^{34}$ These effects of anacetrapib on lipoprotein metabolism contrast with its demonstrated reduction in Lp(a) production. ${ }^{35}$ Accordingly, these metabolic studies have provided important insights into the impact of CETP inhibition on lipoprotein metabolism.

\section{Effect on lipid parameters in clinical studies}

Multiple studies have consistently demonstrated that potent CETP inhibition produces robust changes in lipid parameters, including HDL-C elevation by more than $130 \%$, reductions in LDL-C by $35 \%-40 \%$ and $\mathrm{Lp}$ (a) by $25 \%$ and increases in systemic cholesterol efflux capacity and antiinflammatory potential of HDL. ${ }^{24}$ These lipid effects have been demonstrated across a broad range of cardiovascular risk and baseline lipid levels. The Randomized Evaluation of Anacetrapib Lipid-Modifying Therapy in Patients with Heterozygous Familial Hypercholesterolemia (REALIZE) study evaluated the impact of treatment with anacetrapib for 52 weeks in patients with either genetically or clinically diagnosed heterozygous familial hypercholesterolemia with baseline LDL-C levels greater than treatment targets. This study demonstrated a $36 \%$ reduction in LDL-C, suggesting that CETP inhibition might provide an additional therapeutic approach to lipid lowering in patients who remain at unacceptably high LDL-C levels, despite use of established medical therapies. ${ }^{36}$

The Determining the Efficacy and Tolerability of CETP Inhibition with Anacetrapib (DEFINE) study evaluated the 
efficacy and safety of longer-term administration of anacetrapib in 1,623 patients with high vascular risk. This study was performed following the termination of the torcetrapib program, enabling the opportunity to establish the risk of demonstrating a similar degree of clinical toxicity. Bayesian analysis determined that there was a 94\% likelihood that anacetrapib did not possess such effects, which was reassuring and gave the impetus for embarking on a large cardiovascular outcomes trial. A reduction in adjudicated cardiovascular events, predominantly due to less coronary revascularization procedures, was observed in this study. ${ }^{24}$ Subsequent analysis revealed persistent lipid changes following cessation of anacetrapib administration, which led to more extensive characterization of adipose tissue distribution and a long terminal half-life of anacetrapib, which was found to be detectable in the circulation for several years. ${ }^{37}$

\section{Effect on cardiovascular outcomes}

The Randomized Evaluation of the Effects of Anacetrapib through Lipid Modification (REVEAL) study evaluated the impact of anacetrapib on cardiovascular events in high-risk patients. In this clinical trial, 30,449 patients with established atherosclerotic cardiovascular disease (myocardial infarction, cerebrovascular atherosclerotic disease, peripheral arterial disease or diabetes with symptomatic coronary disease and no acute event in the last 3 months) greater than 50 years of age were treated with anacetrapib $100 \mathrm{mg}$ daily or matching placebo daily for a median follow-up period of 4.1 years. At baseline, mean levels of LDL-C of $61 \mathrm{mg} / \mathrm{dL}$, non-HDL-C of $92 \mathrm{mg} / \mathrm{dL}$ and HDL-C of $40 \mathrm{mg} / \mathrm{dL}$ were observed, suggesting that patients were well treated by contemporary standards. $^{38}$

Compared with placebo, anacetrapib-treated patients demonstrated a higher level of HDL-C by $104 \%$ and lower level of non-HDL-C by $18 \%$. The incidence of the composite primary end point of coronary death, myocardial infarction or coronary revascularization was reduced by $9 \%$ in the anacetrapib group (10.8\% vs $11.8 \%, P=0.004)$. Anacetrapib was associated with significant reductions in myocardial infarction by $13 \%(6.1 \%$ vs $6.9 \%, P=0.007)$ and coronary revascularization by $10 \%(7.1 \%$ vs $7.9 \%, P=0.01)$, and no reduction in coronary death $(2.5 \%$ vs $2.8 \%, P=0.25)$ or ischemic stroke (3.2\% vs $3.2 \%)$. Importantly, it took greater than 2 years before there was any separation of the event curves. Consistent with prior observations of CETP inhibitors, anacetrapib treatment was associated with a lower incidence of new-onset diabetes mellitus (5.3\% vs $6.0 \%$,
$P=0.0496)$. There were no significant differences between the groups with regard to the risk of cancer or other serious adverse events. ${ }^{38}$

This is an important finding. For the first time, a large cardiovascular outcomes trial demonstrated that administration of a potent CETP inhibitor produced a reduction in major adverse cardiovascular events. This finding was observed in a clinical trial incorporating a larger study cohort with much longer treatment duration than employed in outcomes trials of previous CETP inhibitors. Subsequent analysis has demonstrated that the degree of reduction in clinical events associated with the decrease in non-HDL-C, confirming a similar relationship in other lipid-lowering trials. ${ }^{38}$ The results are consistent with reports from Mendelian randomization studies that predicted that pharmacological inhibition of CETP would result in a cardiovascular event reduction by $8 \%-12 \% .{ }^{39}$ Of particular interest, these genetic studies suggest potential discord between lowering of LDL-C and apoB and CETP inhibitors in the setting of concomitant statin therapy, suggesting potentially greater benefit of CETP inhibition when administered as monotherapy. ${ }^{39}$ The impact of this form of administration has not yet been evaluated in large clinical outcomes trial. The results of REVEAL were also observed in a cohort who were very well treated established cardioprotective agents. Whether a greater benefit would have been demonstrated in a cohort with less optimally controlled LDL-C at baseline remains to be determined.

The next question in the anacetrapib story will be whether this agent is translated to clinical practice. While anacetrapib did result in a significant reduction in cardiovascular events, the $9.1 \%$ decrease is relatively modest. It is uncertain whether this benefit will be deemed sufficient to warrant introduction of a new therapeutic. Further analysis that determines the cost effectiveness of this agent will be important in guiding such decisions. Whether the long terminal half-life of anacetrapib, which to date has not been demonstrated to produce any concerning safety issues, will influence the ability to use it in clinical practice also remains to be established.

\section{Summary and future directions}

For two decades, there has been considerable interest in the development of CETP inhibition as an approach to cardiovascular prevention. Despite sequential disappointments in number of development programs, the ability to translate favorable effects of anacetrapib on lipid parameters to cardiovascular events provides hope that CETP inhibition will be of use in the clinic. Whether the long-term adipose 
accumulation of this agent has contributed to the decision not to pursue regulatory approval for use of anacetrapib remains to be determined. Similarly, whether an additional potent CETP inhibitor, TA-8995, will proceed in clinical development is unknown. Regardless, these studies have shed new light on the potential of CETP inhibitors to provide clinical benefit for patients with high vascular risk.

\section{Disclosure}

SJN has received research support from AstraZeneca, Amgen, Anthera, Eli Lilly, Esperion, Novartis, Cerenis, The Medicines Company, Resverlogix, Infraredx, Roche, Sanofi-Regeneron and LipoScience, and is a consultant for AstraZeneca, Eli Lilly, Anthera, Omthera, Merck, Takeda, Resverlogix, Sanofi-Regeneron, CSL Behring, Esperion and Boehringer Ingelheim. BAD reports no conflicts of interest in this work.

\section{References}

1. Barter PJ, Chapman MJ, Hennekens CH, Rader DJ, Tall AR. Cholesteryl ester transfer protein. A novel target for raising HDL and inhibiting atherosclerosis. Arterioscler Thromb Vasc Biol. 2003;23(2):160-167.

2. Brousseau ME, Schaefer EJ, Wolfe ML, et al. Effects of an inhibitor of cholesteryl ester transfer protein on HDL cholesterol. N Engl J Med. 2004;350(15):1505-1515.

3. Rittershaus CW, Miller DP, Thomas LJ, et al. Vaccine-induced antibodies inhibit CETP activity in vivo and reduce aortic lesions in a rabbit model of atherosclerosis. Arterioscler Thromb Vasc Biol. 2000; 20(9):2106-2112.

4. Sugano M, Makino N, Sawada S, et al. Effect of antisense oligonucleotides against cholesteryl ester transfer protein on the development of atherosclerosis in cholesterol-fed rabbits. J Biol Chem. 1998;273(9): 5033-5036.

5. Okamoto H, Yonemori F, Wakitani K, Minowa T, Maeda K, Shinkai H. A cholesteryl ester transfer protein inhibitor attenuates atherosclerosis in rabbits. Nature. 2000;406(6792):203-207.

6. Morehouse LA, Sugarman ED, Bourassa PA, et al. Inhibition of CETP activity by torcetrapib reduces susceptibility to diet-induced atherosclerosis in New Zealand White rabbits. J Lipid Res. 2007;48(6): 1263-1272.

7. Thompson A, Di Angelantonio E, Sarwar N, et al. Association of cholesteryl ester transfer protein genotypes with CETP mass and activity, lipid levels, and coronary risk. JAMA. 2008;299(23):2777-2788.

8. Johannsen TH, Frikke-Schmidt R, Schou J, Nordestgaard BG, TybjaergHansen A. Genetic inhibition of CETP, ischemic vascular disease and mortality, and possible adverse effects. J Am Coll Cardiol. 2012;60(20): 2041-2048.

9. Barter PJ, Caulfield M, Eriksson M, et al; ILLUMINATE Investigators. Effects of torcetrapib in patients at high risk for coronary events. N Engl J Med. 2007;357(21):2109-2122.

10. Nissen SE, Tardif JC, Nicholls SJ, et al; ILLUSTRATE Investigators. Effect of torcetrapib on the progression of coronary atherosclerosis. N Engl J Med. 2007;356(13):1304-1316.

11. Bots ML, Visseren FL, Evans GW, et al; RADIANCE 2 Investigators. Torcetrapib and carotid intima-media thickness in mixed dyslipidaemia (RADIANCE 2 study): a randomised, double-blind trial. Lancet. 2007; 370(9582):153-160.
12. Kastelein JJ, van Leuven SI, Burgess L, et al; RADIANCE 1 Investigators. Effect of torcetrapib on carotid atherosclerosis in familial hypercholesterolemia. $N$ Engl J Med. 2007;356(16):1620-1630.

13. Yvan-Charvet L, Matsuura F, Wang N, et al. Inhibition of cholesteryl ester transfer protein by torcetrapib modestly increases macrophage cholesterol efflux to HDL. Arterioscler Thromb Vasc Biol. 2007;27(5): $1132-1138$.

14. Nicholls SJ, Tuzcu EM, Brennan DM, Tardif JC, Nissen SE. Cholesteryl ester transfer protein inhibition, high-density lipoprotein raising, and progression of coronary atherosclerosis: insights from ILLUSTRATE (Investigation of Lipid Level Management Using Coronary Ultrasound to Assess Reduction of Atherosclerosis by CETP Inhibition and HDL Elevation). Circulation. 2008;118(24):2506-2514.

15. Barter P. Lessons learned from the Investigation of Lipid Level Management to Understand its Impact in Atherosclerotic Events (ILLUMINATE) trial. Am J Cardiol. 2009;104(10 Suppl):10E-15E.

16. Vergeer M, Stroes ES. The pharmacology and off-target effects of some cholesterol ester transfer protein inhibitors. Am J Cardiol. 2009; 104(10 Suppl):32E-38E.

17. Lüscher TF, Taddei S, Kaski JC, et al; dal-VESSEL Investigators. Vascular effects and safety of dalcetrapib in patients with or at risk of coronary heart disease: the dal-VESSEL randomized clinical trial. Eur Heart J. 2012;33(7):857-865.

18. Fayad ZA, Mani V, Woodward M, et al; dal-PLAQUE Investigators. Safety and efficacy of dalcetrapib on atherosclerotic disease using novel non-invasive multimodality imaging (dal-PLAQUE): a randomised clinical trial. Lancet. 2011;378(9802):1547-1559.

19. Schwartz GG, Olsson AG, Abt M, et al; dal-OUTCOMES Investigators. Effects of dalcetrapib in patients with a recent acute coronary syndrome. N Engl J Med. 2012;367(22):2089-2099.

20. Tardif JC, Rhéaume E, Lemieux Perreault LP, et al. Pharmacogenomic determinants of the cardiovascular effects of dalcetrapib. Circ Cardiovasc Genet. 2015;8(2):372-382.

21. Nicholls SJ, Brewer HB, Kastelein JJ, et al. Effects of the CETP inhibitor evacetrapib administered as monotherapy or in combination with statins on HDL and LDL cholesterol: a randomized controlled trial. JAMA. 2011;306(19):2099-2109.

22. Lincoff AM, Nicholls SJ, Riesmeyer JS, et al; ACCELERATE Investigators. Evacetrapib and cardiovascular outcomes in high-risk vascular disease. N Engl J Med. 2017;376(20):1933-1942.

23. Nicholls SJ, Ray KK, Ballantyne CM, et al; ACCENTUATE Investigators. Comparative effects of cholesteryl ester transfer protein inhibition, statin or ezetimibe on lipid factors: the ACCENTUATE trial. Atherosclerosis. 2017;261:12-18.

24. Cannon CP, Shah S, Dansky HM, et al; Determining the Efficacy and Tolerability Investigators. Safety of anacetrapib in patients with or at high risk for coronary heart disease. $N$ Engl J Med. 2010;363(25): 2406-2415.

25. Forrest MJ, Bloomfield D, Briscoe RJ, et al. Torcetrapib-induced blood pressure elevation is independent of CETP inhibition and is accompanied by increased circulating levels of aldosterone. Br J Pharmacol. 2008;154(7):1465-1473.

26. Kühnast S, van der Tuin SJ, van der Hoorn JW, et al. Anacetrapib reduces progression of atherosclerosis, mainly by reducing non-HDLcholesterol, improves lesion stability and adds to the beneficial effects of atorvastatin. Eur Heart J. 2015;36(1):39-48.

27. Simic B, Mocharla P, Crucet M, et al. Anacetrapib, but not evacetrapib, impairs endothelial function in CETP-transgenic mice in spite of marked HDL-C increase. Atherosclerosis. 2017;257:186-194.

28. Krishna R, Gheyas F, Liu Y, et al. Chronic administration of anacetrapib is associated with accumulation in adipose and slow elimination. Clin Pharmacol Ther. 2017;102(5):832-840.

29. Castro-Perez J, Briand F, Gagen K, et al. Anacetrapib promotes reverse cholesterol transport and bulk cholesterol excretion in Syrian golden hamsters. J Lipid Res. 2011;52(11):1965-1973. 
30. Wu BJ, Shrestha S, Ong KL, et al. Cholesteryl ester transfer protein inhibition enhances endothelial repair and improves endothelial function in the rabbit. Arterioscler Thromb Vasc Biol. 2015;35(3):628-636.

31. Wu BJ, Shrestha S, Ong KL, et al. Increasing HDL levels by inhibiting cholesteryl ester transfer protein activity in rabbits with hindlimb ischemia is associated with increased angiogenesis. Int J Cardiol. 2015;199:204-212.

32. Reyes-Soffer G, Millar JS, Ngai C, et al. Cholesteryl ester transfer protein inhibition with anacetrapib decreases fractional clearance rates of high-density lipoprotein apolipoprotein A-I and plasma cholesteryl ester transfer protein. Arterioscler Thromb Vasc Biol. 2016;36(5): 994-1002.

33. Millar JS, Reyes-Soffer G, Jumes P, et al. Anacetrapib lowers LDL by increasing ApoB clearance in mildly hypercholesterolemic subjects. $J$ Clin Invest. 2015;125(6):2510-2522.

34. Millar JS, Lassman ME, Thomas T, et al. Effects of CETP inhibition with anacetrapib on metabolism of VLDL-TG and plasma apolipoproteins C-II, C-III, and E. J Lipid Res. 2017;58(6):1214-1220.
35. Thomas T, Zhou H, Karmally W, et al. CETP (cholesteryl ester transfer protein) inhibition with anacetrapib decreases production of lipoprotein(a) in mildly hypercholesterolemic subjects. Arterioscler Thromb Vasc Biol. 2017;37(9):1770-1775.

36. Kastelein JJ, Besseling J, Shah S, et al. Anacetrapib as lipid-modifying therapy in patients with heterozygous familial hypercholesterolaemia (REALIZE): a randomised, double-blind, placebo-controlled, phase 3 study. Lancet. 2015;385(9983):2153-2161.

37. Gotto AM Jr, Kher U, Chatterjee MS, et al; DEFINE Investigators. Lipids, safety parameters, and drug concentrations after an additional 2 years of treatment with anacetrapib in the DEFINE study. J Cardiovasc Pharmacol Ther. 2014;19(6):543-549.

38. HPS3/TIMI55-REVEAL Collaborative Group, Bowman L, Hopewell JC, Chen $\mathrm{F}$, et al. Effects of anacetrapib in patients with atherosclerotic vascular disease. N Engl J Med. 2017;377(13):1217-1227.

39. Ference BA, Kastelein JJP, Ginsberg HN, et al. Association of genetic variants related to CETP inhibitors and statins with lipoprotein levels and cardiovascular risk. JAMA. 2017;318(10):947-956.

\section{Publish your work in this journal}

Drug Design, Development and Therapy is an international, peerreviewed open-access journal that spans the spectrum of drug design and development through to clinical applications. Clinical outcomes, patient safety, and programs for the development and effective, safe, and sustained use of medicines are the features of the journal, which has also been accepted for indexing on PubMed Central. The manuscript management system is completely online and includes a very quick and fair peer-review system, which is all easy to use. Visit http://www.dovepress.com/testimonials.php to read real quotes from published authors.

Submit your manuscript here: http://www.dovepress.com/drug-design-development-and-therapy-journal 